\title{
ON THE RINGS OF FRICKE CHARACTERS OF FREE ABELIAN GROUPS
}

\author{
ERI HATAKENAKA AND TAKAO SATOH
}

\begin{abstract}
In this paper, we determine the structure of the rings $\mathfrak{X}_{\mathbf{Q}}(H)$ of Fricke characters over $\mathbf{Q}$ of free abelian groups $H$ of rank $n \geq 1$. In particular, we consider the ideal $J$ in $\mathfrak{X}_{\mathbf{Q}}(H)$, generated by $\operatorname{tr} x-2$ for any $x \in H$ and give a Q-basis of each of the graded quotients $\operatorname{gr}^{k}(J):=J^{k} / J^{k+1}$ for $k \geq 1$. Then we introduce a weight for each element of $\mathfrak{X}_{\mathbf{Q}}(H)$. By using the concept of this weight we show that $\mathfrak{X}_{\mathbf{Q}}(H)$ is an integral domain.
\end{abstract}

1. Introduction. Let $G$ be a group generated by $x_{1}, \ldots, x_{n}$. We denote by $R(G)$ the set $\operatorname{Hom}(G$, SL $(2, \mathbf{C}))$ of all SL $(2, \mathbf{C})$-representations of $G$. Let $\mathcal{F}(R(G), \mathbf{C})$ be the set $\{\chi: R(G) \rightarrow \mathbf{C}\}$ of all complex-valued functions on $R(G)$. Then we can regard $\mathcal{F}(R(G), \mathbf{C})$ to be a $\mathbf{C}$-algebra in a natural way. (See subsection 3.1 for details.) For an element $x \in G$, $\operatorname{tr} x \in \mathcal{F}(R(G), \mathbf{C})$ is defined to satisfy the equation

$$
(\operatorname{tr} x)(\rho)=\operatorname{tr} \rho(x)
$$

for any $\rho \in R(G)$. Here "tr" in the right hand side means the trace of $2 \times 2$ matrix $\rho(x) \in \mathrm{SL}(2, \mathbf{C})$. The element $\operatorname{tr} x$ in $\mathcal{F}(R(G), \mathbf{C})$ is called the Fricke character of $x$. Let $\mathfrak{X}_{\mathbf{Q}}(G)$ be the $\mathbf{Q}$-vector subspace in $\mathcal{F}(R(G), \mathbf{C})$, which is generated by all $\operatorname{tr} x$ for $x \in G$. Then $\mathfrak{X}_{\mathbf{Q}}(G)$ has a ring structure with the multiplication of $\mathcal{F}(R(G), \mathbf{C})$. We call $\mathfrak{X}_{\mathbf{Q}}(G)$ the ring of Fricke characters of $G$ over $\mathbf{Q}$.

Classically, the study of Fricke characters was begun by Fricke for a free group $F_{n}$ generated by $x_{1}, \ldots, x_{n}$ in connection with certain

2010 AMS Mathematics subject classification. Primary 20G05, Secondary $13 \mathrm{~A} 15,13 \mathrm{C} 05$.

Keywords and phrases. Fricke characters, SL $(2, \mathbf{C})$-trace formula, character variety.

The second author is supported by a Grant-in-Aid for Young Scientists (B) by JSPS.

Received by the editors on March 4, 2013, and in revised form on September 7, 2014. 
problems in the theory of Riemann surfaces. (See [3].) In the 1970s, Horowitz investigated algebraic properties of $\mathfrak{X}(G)$ by using the combinatorial group theory in $[6,7]$. In particular, he showed in [6] that, for any $x \in G$, the Fricke character $\operatorname{tr} x$ can be written as a rational polynomial in $n+\left(\begin{array}{c}n \\ 2\end{array}\right)+\left(\begin{array}{l}n \\ 3\end{array}\right)$ characters $\operatorname{tr} x_{i_{1}} x_{i_{2}} \cdots x_{i_{l}}$ for $1 \leq l \leq 3$ and $1 \leq i_{1}<i_{2}<\cdots<i_{l} \leq n$. Hence, when we put $\mathfrak{P}$ to be a polynomial ring

$$
\mathbf{Q}\left[t_{i_{1} \cdots i_{l}} \mid 1 \leq l \leq 3, \quad 1 \leq i_{1}<i_{2}<\cdots<i_{l} \leq n\right],
$$

then there exists a surjective homomorphism $\pi_{G}: \mathfrak{P} \rightarrow \mathfrak{X}_{\mathbf{Q}}(G)$ defined by $t_{i_{1} \cdots i_{l}} \mapsto \operatorname{tr} x_{i_{1}} x_{i_{2}} \cdots x_{i_{l}}$. The study of the ring structure of $\mathfrak{X}_{\mathbf{Q}}(G)$ is inextricably associated with to that of the ideal $\operatorname{Ker}\left(\pi_{G}\right)$. In general, however, it is quite a difficult problem to find a generating set of $\operatorname{Ker}\left(\pi_{G}\right)$ even in the case that $G$ is a free group. (See also subsection 3.1.)

In the case that $G$ is an abelian group, it is easy to see that any Fricke character of $G$ is written as a rational polynomial in $n+\left(\begin{array}{c}n \\ 2\end{array}\right)$ characters $\operatorname{tr} x_{i}$ for $1 \leq i \leq n$ and $\operatorname{tr} x_{i_{1}} x_{i_{2}}$ for $1 \leq i_{1}<i_{2} \leq n$. Hence, when we put $\mathcal{P}$ as a polynomial ring

$$
\mathbf{Q}\left[t_{i}, t_{i_{1} i_{2}} \mid 1 \leq i \leq n, \quad 1 \leq i_{1}<i_{2} \leq n\right],
$$

then there exists the surjective homomorphism $\bar{\pi}_{G}: \mathcal{P} \rightarrow \mathfrak{X}_{\mathbf{Q}}(G)$ defined by $t_{i} \mapsto \operatorname{tr} x_{i}$ and $t_{i_{1} i_{2}} \mapsto \operatorname{tr} x_{i_{1}} x_{i_{2}}$. We denote by $I$ the kernel of $\bar{\pi}_{G}$.

In the present paper, we consider the case where $G$ is a free abelian group $H:=H_{1}\left(F_{n}, \mathbf{Z}\right)$ of rank $n$. First, we introduce a descending filtration in $\mathfrak{X}_{\mathbf{Q}}(H)$. Set $t_{i_{1} \cdots i_{l}}^{\prime}:=t_{i_{1} \cdots i_{l}}-2 \in \mathcal{P}$. We also denote by $t_{i_{1} \ldots i_{l}}^{\prime}$ its coset class in $\mathcal{P} / I \cong \mathfrak{X}_{\mathbf{Q}}(H)$ by abuse of language. Consider the ideal $J_{0}$ in $\mathcal{P}$ generated by all $t_{i_{1} \cdots i_{l}}^{\prime}$ s. That is,

$$
J_{0}=\left(t_{i}^{\prime}, t_{i_{1} i_{2}}^{\prime} \mid 1 \leq i \leq n, 1 \leq i_{1}<i_{2} \leq n\right) \subset \mathcal{P} .
$$

Set $J:=\bar{\pi}_{H}\left(J_{0}\right)$. Then, we have a descending filtration

$$
J \supset J^{2} \supset J^{3} \supset \cdots
$$

of ideals in $\mathcal{P} / I$. (See subsection 3.1 for details.) $\operatorname{Set} \operatorname{gr}^{k}(J):=J^{k} / J^{k+1}$

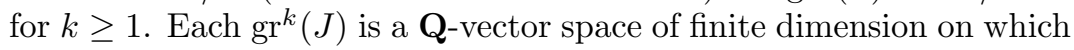
Aut $H$ naturally acts. In general, to determine the structures of the graded quotients $\operatorname{gr}^{k}(J)$ plays an important role on various studies of 
the ring $\mathcal{P} / I$. The first purpose of this paper is to give a basis of $\operatorname{gr}^{k}(J)$ for any $k \geq 1$. More precisely, we show the following theorem.

Theorem 1.1 (equivalent to Theorems 4.2 and 4.5).

(i) For each $k \geq 1$ and $0 \leq l \leq k$, set

$$
\begin{aligned}
T_{l}:= & \left\{t_{p_{1} q_{1}}^{\prime} \cdots t_{p_{l} q_{l}}^{\prime} t_{i_{l+1}}^{\prime} \cdots t_{i_{k}}^{\prime} \in J_{0}\right. \\
& \left.\mid 1 \leq p_{1}<q_{1}<\cdots<p_{l}<q_{l} \leq n, 1 \leq i_{l+1} \leq \cdots \leq i_{k} \leq n\right\} .
\end{aligned}
$$

Then

$$
S_{k}:=\bigcup_{l=0}^{k} \bar{\pi}_{H}\left(T_{l}\right)
$$

(ii)

forms a basis of $g r^{k}(J)$.

$$
\bigcap_{k \geq 1} J^{k}=\{0\} .
$$

The main purpose of this paper is to give an application to our previous work for the Fricke characters of free groups. Let us explain it. Needless to say, by an argument similar to the above, we can define the ideal $J_{F_{n}}$ in the ring $\mathfrak{X}_{\mathbf{Q}}\left(F_{n}\right)$ of Fricke characters of the free group $F_{n}$ generated by all $t_{i_{1} \cdots i_{l}}^{\prime}:=t_{i_{1} \cdots i_{l}}-2$ for $1 \leq l \leq 3$ and $1 \leq i_{1}<i_{2}<$ $\cdots<i_{l} \leq n$. Then we have a descending filtration $J_{F_{n}} \supset J_{F_{n}}^{2} \supset \cdots$ in $\mathfrak{X}_{\mathbf{Q}}\left(F_{n}\right)$ and the graded quotients $\operatorname{gr}^{k}\left(J_{F_{n}}\right):=J_{F_{n}}^{k} / J_{F_{n}}^{k+1}$ for each $k \geq 1$. Such graded quotients were originally studied by Magnus [13] to investigate the behavior of the action of Aut $F_{3}$ on $\operatorname{gr}^{k}\left(J_{F_{n}}\right)$. In [5], we gave bases of the graded quotients $\operatorname{gr}^{k}\left(J_{F_{n}}\right):=J_{F_{n}}^{k} / J_{F_{n}}^{k+1}$ for $k=1$ and 2. In general, however, it seems that no basis of $\operatorname{gr}^{k}\left(J_{F_{n}}\right)$ is obtained for $k \geq 4$. From Theorem 1.1, we can give a lower bound on a dimension of $\operatorname{gr}^{k}\left(J_{F_{n}}\right)$ since the natural projection $F_{n} \rightarrow H$ induces the surjective homomorphism $\operatorname{gr}^{k}\left(J_{F_{n}}\right) \rightarrow \operatorname{gr}^{k}(J)$. More precisely, we have

Corollary 1.2. For any $n \geq 2$,

$$
\operatorname{dim}_{\mathbf{Q}}\left(\operatorname{gr}^{k}\left(J_{F_{n}}\right)\right) \geq \operatorname{dim}_{\mathbf{Q}}\left(\operatorname{gr}^{k}(J)\right)=\sum_{l=0}^{k}\left(\begin{array}{c}
n \\
2 l
\end{array}\right)\left(\begin{array}{c}
n+k-l-1 \\
k-l
\end{array}\right) .
$$


For any $k \geq 1$, let $\mathcal{E}_{F_{n}}(k)$ be the subgroup of Aut $F_{n}$, consisting of automorphisms which act on $J_{F_{n}} / J_{F_{n}}^{k+1}$ trivially. Then we have a descending filtration $\left\{\mathcal{E}_{F_{n}}(k)\right\}_{k \geq 1}$ of Aut $F_{n}$. This filtration is a Fricke character analogue of the Andreadakis-Johnson filtration $\left\{\mathcal{A}_{F_{n}}(k)\right\}_{k \geq 1}$ of Aut $F_{n}$. The Andreadakis-Johnson filtration was originally introduced by Andreadakis [2] in the 1960's. The name "Johnson" comes from Dennis Johnson who studied this type of filtration for the mapping class group of a surface in the 1980's. It is called the Johnson filtration of the mapping class group. The Johnson homomorphisms were originally introduced by Johnson in order to investigate the graded quotients of the Johnson filtration in a series of his pioneering works $[\mathbf{8}, \mathbf{9}, \mathbf{1 0}, \mathbf{1 1}]$. In $[\mathbf{1 4}]$, Morita began to study the Johnson homomorphisms of the mapping class groups and Aut $F_{n}$ systematically. Today, together with the theory of the Johnson homomorphisms, the Andreadakis-Johnson filtration is one of the powerful tools to study the group structure of the automorphism group of a group. (For basic material for the Andreadakis-Johnson filtration and the Johnson homomorphisms of Aut $F_{n}$, see $[\mathbf{1 5}, \mathbf{1 6}]$, for example.) In our previous paper [5], we also studied the graded quotients $\operatorname{gr}^{k}\left(\mathcal{E}_{F_{n}}\right):=\mathcal{E}_{F_{n}}(k) / \mathcal{E}_{F_{n}}(k+1)$, by introducing and using a Johnson homomorphism-like homomorphism

$$
\eta_{k}: \operatorname{gr}^{k}\left(\mathcal{E}_{F_{n}}\right) \longrightarrow \operatorname{Hom}_{\mathbf{Q}}\left(\operatorname{gr}^{1}\left(J_{F_{n}}\right), \operatorname{gr}^{k+1}\left(J_{F_{n}}\right)\right)
$$

for each $k \geq 1$. In general, to determine the image of $\eta_{k}$ is quite a difficult problem. Since $\eta_{k}$ vanishes through the homomorphism

$$
\operatorname{Hom}_{\mathbf{Q}}\left(\operatorname{gr}^{1}\left(J_{F_{n}}\right), \operatorname{gr}^{k+1}\left(J_{F_{n}}\right)\right) \longrightarrow \operatorname{Hom}_{\mathbf{Q}}\left(\operatorname{gr}^{1}\left(J_{F_{n}}\right), \operatorname{gr}^{k+1}(J)\right)
$$

induced from the homomorphism $\operatorname{gr}^{k+1}\left(J_{F_{n}}\right) \rightarrow \operatorname{gr}^{k+1}(J)$ obtained from the natural projection $F_{n} \rightarrow H$, we can obtain information about the cokernel of $\eta_{k}$ by Theorem 1.1.

Corollary 1.3. For any $n \geq 3$ and $k \geq 2$,

$$
\operatorname{dim}_{\mathbf{Q}}\left(\operatorname{Coker}\left(\eta_{k}\right)\right) \geq\left(n+\left(\begin{array}{l}
n \\
2
\end{array}\right)+\left(\begin{array}{l}
n \\
3
\end{array}\right)\right) \sum_{l=0}^{k+1}\left(\begin{array}{l}
n \\
2 l
\end{array}\right)\left(\begin{array}{l}
n+k-l \\
k+1-l
\end{array}\right) .
$$

In [5], we also showed that $\mathcal{A}_{F_{n}}(2 k) \subset \mathcal{E}_{F_{n}}(k)$ for each $k \geq 1$. Hence, the natural inclusion map and $\eta_{k}$ define the homomorphism

$$
\mathcal{A}_{F_{n}}(2 k) / \mathcal{A}_{F_{n}}(2 k+2) \rightarrow \operatorname{gr}^{k}\left(\mathcal{E}_{F_{n}}\right) \stackrel{\eta_{k}}{\longrightarrow} \operatorname{Hom}_{\mathbf{Q}}\left(\operatorname{gr}^{1}\left(J_{F_{n}}\right), \operatorname{gr}^{k+1}\left(J_{F_{n}}\right)\right)
$$


for each $k \geq 1$. At the present stage, we do not know whether the above homomorphism is injective or not. To the best of our knowledge, other than this, it seems quite difficult to develop the connection of the ring of Fricke characters to the Lie algebra arising from the AndreadakisJohnson filtration.

Now, by using Theorem 1.1, we can obtain the following.

Theorem 1.4 (equivalent to Theorem 4.3). The ideal I is generated by

$$
\begin{aligned}
t_{i r}^{\prime} t_{j s}^{\prime} & -t_{i s}^{\prime} t_{j r}^{\prime}-\left\{t_{i}^{\prime} t_{r}^{\prime}+t_{j}^{\prime} t_{s}^{\prime}-t_{j}^{\prime} t_{r}^{\prime}-t_{i}^{\prime} t_{s}^{\prime}\right\} \\
& +\left\{t_{i}^{\prime} t_{j r}^{\prime}+t_{j}^{\prime} t_{i s}^{\prime}+t_{r}^{\prime} t_{i s}^{\prime}+t_{s}^{\prime} t_{j r}^{\prime}-t_{j}^{\prime} t_{i r}^{\prime}-t_{i}^{\prime} t_{j s}^{\prime}-t_{r}^{\prime} t_{j s}^{\prime}-t_{s}^{\prime} t_{i r}^{\prime}\right\} \\
& +\frac{1}{2}\left\{t_{j}^{\prime} t_{r}^{\prime} t_{i s}^{\prime}+t_{i}^{\prime} t_{s}^{\prime} t_{j r}^{\prime}-t_{i}^{\prime} t_{r}^{\prime} t_{j s}^{\prime}-t_{j}^{\prime} t_{s}^{\prime} t_{i r}^{\prime}\right\}
\end{aligned}
$$

for any $1 \leq i, j, r, s \leq n$. Here we remark that, in the above notation, $t_{i j}^{\prime}$ should be read

$$
\begin{cases}t_{j i}^{\prime} & \text { if } i>j, \\ \left(t_{i}^{\prime}\right)^{2}+4 t_{i}^{\prime} & \text { if } i=j .\end{cases}
$$

In particular, I is finitely generated.

Furthermore, from Theorem 1.1, we see that the ideal $I$ is generated by polynomials of degree greater than one. This fact enables us to define the concept of a weight for each element of $\mathcal{P} / I \cong \mathfrak{X}_{\mathbf{Q}}(H)$. Using this, we show

Theorem 1.5 (equivalent to Theorem 5.2). The ring $\mathfrak{X}_{\mathbf{Q}}(H)$ of Fricke characters is an integral domain. That is, the ideal $I$ is a prime ideal in $\mathcal{P}$.

Finally, in Section 6, we will give some remarks on the natural action of Aut $H$ on $J / J^{k+1}$ for $k \geq 1$. Let $\mathcal{E}_{H}(k)$ be the kernel of a natural homomorphism Aut $H \rightarrow \operatorname{Aut}\left(J / J^{k+1}\right)$ induced from the action of Aut $G$. Let $\iota \in$ Aut $H$ be an automorphism of $H$ defined by

$$
x_{i}^{\iota}:=x_{i}^{-1}, \quad 1 \leq i \leq n .
$$

Then we show the following. 
Proposition 1.6 (equivalent to Proposition 6.1 and Corollary 6.2). For any $k \geq 1$, the group $\mathcal{E}_{H}(k)$ is the cyclic group of order 2 , generated by $\iota$.

2. Notation and conventions. Throughout the paper, we use the following notation and conventions. Let $F_{n}$ be the free group of rank $n$ with a basis $x_{1}, \ldots, x_{n}$, and let $H$ be its abelianization $H_{1}\left(F_{n}, \mathbf{Z}\right)$. Then $H$ is a free abelian group of rank $n$, and the coset classes of $x_{1}, \ldots, x_{n}$ form a basis of $H$ as a free abelian group. We also use the following notation.

- Let $G$ be a group. The automorphism group Aut $G$ of $G$ acts on $G$ from the right. For any $\sigma \in$ Aut $G$ and $x \in G$, the action of $\sigma$ on $x$ is denoted by $x^{\sigma}$.

- Let $N$ be a normal subgroup of a group $G$. For an element $g \in G$, we also denote the coset class of $g$ by $g \in G / N$ if there is no confusion. Similarly, for a ring $R$, an element $f \in R$ and an ideal $I$ of $R$, we also denote by $f$ the coset class of $f$ in $R / I$ if there is no confusion.

- For elements $x$ and $y$ in $G$, the commutator bracket $[x, y]$ of $x$ and $y$ is defined to be $x y x^{-1} y^{-1}$.

For pairs $\left(i_{1}, i_{2}, \ldots, i_{k}\right)$ and $\left(j_{1}, j_{2}, \ldots, j_{k}\right)$ of natural numbers $i_{r}, j_{s} \in$ $\mathbf{N}$, we denote the lexicographic order among them by $\left(i_{1}, i_{2}, \ldots, i_{k}\right) \leq$ $\left(j_{1}, j_{2}, \ldots, j_{k}\right)$. Namely, this means $i_{1}<j_{1}, i_{1}=j_{1}$ and $i_{2}<j_{2}$, or and so on.

3. A filtration on the ring of Fricke characters of $H$. In this section, we briefly review the ring of Fricke characters of a finitely generated group $G$.

3.1. The ring $\mathfrak{X}_{\mathbf{Q}}(G)$ of Fricke characters of a finitely generated group $G$. Let $G$ be a group generated by $x_{1}, \ldots, x_{n}$. We denote by $R(G)$ the set $\operatorname{Hom}(G, \operatorname{SL}(2, \mathbf{C}))$ of all SL $(2, \mathbf{C})$-representations of $G$. Let $\mathcal{F}(R(G), \mathbf{C})$ be the set $\{\chi: R(G) \rightarrow \mathbf{C}\}$ of all complex-valued functions on $R(G)$. Then $\mathcal{F}(R(G), \mathbf{C})$ has a $\mathbf{C}$-algebra structure by the operations defined by

$$
\begin{aligned}
\left(\chi+\chi^{\prime}\right)(\rho) & :=\chi(\rho)+\chi^{\prime}(\rho), \\
\left(\chi \chi^{\prime}\right)(\rho) & :=\chi(\rho) \chi^{\prime}(\rho),
\end{aligned}
$$




$$
(\lambda \chi)(\rho):=\lambda(\chi(\rho)),
$$

for any $\chi, \chi^{\prime} \in \mathcal{F}(R(G), \mathbf{C}), \lambda \in \mathbf{C}$ and $\rho \in R(G)$.

The automorphism group Aut $G$ of $G$ naturally acts on $R(G)$ and $\mathcal{F}(R(G), \mathbf{C})$ from the right by

$$
\rho^{\sigma}(x):=\rho\left(x^{\sigma^{-1}}\right), \quad \rho \in R(G) \quad \text { and } \quad x \in G
$$

and

$$
\chi^{\sigma}(\rho):=\chi\left(\rho^{\sigma^{-1}}\right), \quad \chi \in \mathcal{F}(R(G), \mathbf{C}) \quad \text { and } \quad \rho \in R(G),
$$

for any $\sigma \in \operatorname{Aut} G$.

For any $x \in G$, we define an element $\operatorname{tr} x$ of $\mathcal{F}(R(G), \mathbf{C})$ to be

$$
(\operatorname{tr} x)(\rho):=\operatorname{tr} \rho(x),
$$

for any $\rho \in R(G)$. Here, "tr" in the right hand side means the trace of the $2 \times 2$ matrix $\rho(x)$. The element $\operatorname{tr} x$ in $\mathcal{F}(R(G), \mathbf{C})$ is called the Fricke character of $x \in G$. The action of an element $\sigma \in \operatorname{Aut} G$ on $\operatorname{tr} x$ is given by $\operatorname{tr} x^{\sigma}$. We have the following well-known formulae:

$$
\operatorname{tr} x^{-1}=\operatorname{tr} x
$$

$$
\operatorname{tr} x y=\operatorname{tr} y x
$$

$$
\operatorname{tr} x y+\operatorname{tr} x y^{-1}=(\operatorname{tr} x)(\operatorname{tr}),
$$

$$
\begin{aligned}
\operatorname{tr} x y z+\operatorname{tr} y x z & =(\operatorname{tr} x)(\operatorname{tr} y z)+(\operatorname{tr} y)(\operatorname{tr} x z) \\
& +(\operatorname{tr} z)(\operatorname{tr} x y)-(\operatorname{tr} x)(\operatorname{tr} y)(\operatorname{tr} z),
\end{aligned}
$$

$$
\operatorname{tr}[x, y]=(\operatorname{tr} x)^{2}+(\operatorname{tr} y)^{2}+(\operatorname{tr} x y)^{2}-(\operatorname{tr} x)(\operatorname{tr} y)(\operatorname{tr} x y)-2,
$$

$$
\begin{aligned}
& 2 \operatorname{tr} x y z w=(\operatorname{tr} x)(\operatorname{tr} y z w)+(\operatorname{tr} y)(\operatorname{tr} z w x)+(\operatorname{tr} z)(\operatorname{tr} w x y) \\
& \quad+(\operatorname{tr} w)(\operatorname{tr} x y z)+(\operatorname{tr} x y)(\operatorname{tr} z w)-(\operatorname{tr} x z)(\operatorname{tr} y w)+(\operatorname{tr} x w)(\operatorname{tr} y z) \\
& \quad-(\operatorname{tr} x)(\operatorname{tr} y)(\operatorname{tr} z w)-(\operatorname{tr} y)(\operatorname{tr} z)(\operatorname{tr} x w)-(\operatorname{tr} x)(\operatorname{tr} w)(\operatorname{tr} y z) \\
& \quad-(\operatorname{tr} z)(\operatorname{tr} w)(\operatorname{tr} x y)+(\operatorname{tr} x)(\operatorname{tr} y)(\operatorname{tr} z)(\operatorname{tr} w),
\end{aligned}
$$


for any $x, y, z, w \in G$. The equations (3.4) and (3.6) are due to Vogt [17]. (For details, see [12, subsection 3.4], for example.)

Let $\mathfrak{X}_{\mathbf{Q}}(G)$ be the $\mathbf{Q}$-vector subspace of $\mathcal{F}(R(G), \mathbf{C})$ generated by all tr $x$ for $x \in G$. The set $\mathfrak{X}_{\mathbf{Q}}(G)$ naturally has a ring structure from (3.3). We call $\mathfrak{X}_{\mathbf{Q}}(G)$ the ring of Fricke characters of $G$ over $\mathbf{Q}$. Let $\mathfrak{P}$ be a rational polynomial ring

$$
\mathbf{Q}\left[t_{i_{1} \cdots i_{l}} \mid 1 \leq l \leq 3,1 \leq i_{1}<i_{2}<\cdots<i_{l} \leq n\right]
$$

of $n+\left(\begin{array}{l}n \\ 2\end{array}\right)+\left(\begin{array}{l}n \\ 3\end{array}\right)$ indeterminates. Consider a ring homomorphism $\pi=\pi_{G}: \mathfrak{P} \rightarrow \mathcal{F}(R(G), \mathbf{C})$ defined by

$$
\pi(1):=\frac{1}{2}\left(\operatorname{tr} 1_{G}\right), \quad \pi\left(t_{i_{1} \cdots i_{l}}\right):=\operatorname{tr} x_{i_{1}} \cdots x_{i_{l}} .
$$

We see $\operatorname{Im}(\pi) \subset \mathfrak{X}_{\mathbf{Q}}(G)$. By a classical result due to Horowitz, we have

Theorem 3.1 (Horowitz, $[6]$ ). For any group $G$ generated by $x_{1}, \ldots, x_{n}$, the homomorphism $\pi: \mathfrak{P} \rightarrow \mathfrak{X}_{\mathbf{Q}}(G)$ is surjective.

More precisely, Horowitz obtained a generating set of the ring of Fricke characters of $G$ over $\mathbf{Z}$ in [6]. Using this and (3.6), we can obtain the above theorem. We should remark that, in general, the structure of an ideal

$$
\operatorname{Ker}\left(\pi_{G}\right)=\left\{f \in \mathfrak{P} \mid f\left(\operatorname{tr} \rho\left(x_{i_{1}} \cdots x_{i_{l}}\right)\right)=0 \quad \text { for any } \rho \in R(G)\right\}
$$

is quite difficult. For example, its generating set is not obtained even in the case where $G$ is a free group $F_{n}$ in general. Horowitz [6] showed that $\operatorname{Ker}\left(\pi_{F_{n}}\right)=(0)$ for $n=1$ and 2 , and that $\operatorname{Ker}\left(\pi_{F_{3}}\right)$ is a principal ideal generated by a quadratic polynomial

$$
t_{123}^{2}-P_{123}(t) t_{123}+Q_{123}(t),
$$

where

$$
\begin{aligned}
P_{a b c}(t):= & t_{a b} t_{c}+t_{a c} t_{b}+t_{b c} t_{a}-t_{a} t_{b} t_{c}, \\
Q_{a b c}(t):= & t_{a}^{2}+t_{b}^{2}+t_{c}^{2}+t_{a b}^{2}+t_{a c}^{2}+t_{b c}^{2}-t_{a} t_{b} t_{a b} \\
& -t_{a} t_{c} t_{a c}-t_{b} t_{c} t_{b c}+t_{a b} t_{b c} t_{a c}-4 .
\end{aligned}
$$

For $n \geq 4$, Whittemore [18] showed that the ideal $\operatorname{Ker}\left(\pi_{F_{n}}\right)$ is not principal. However, very little is known about $\operatorname{Ker}\left(\pi_{F_{n}}\right)$ for general $n \geq 4$. 
3.2. The ring $\mathfrak{X}_{\mathbf{Q}}(G)$ for an abelian group $G$. Now, in the following, we always consider the case where $G$ is abelian. In this case, we see

$$
2 \operatorname{tr} x y z=(\operatorname{tr} x)(\operatorname{tr} y z)+(\operatorname{tr} y)(\operatorname{tr} x z)+(\operatorname{tr} z)(\operatorname{tr} x y)-(\operatorname{tr} x)(\operatorname{tr} y)(\operatorname{tr} z),
$$

from (3.4). This shows that, if $G$ is abelian and is generated by $x_{1}, \ldots, x_{n}$, it turns out that $\mathfrak{X}_{\mathbf{Q}}(G)$ is generated by $\operatorname{tr} 1_{G}$,

$$
\operatorname{tr} x_{i} \text { for } 1 \leq i \leq n \text { and } \operatorname{tr} x_{i} x_{j} \text { for } 1 \leq i<j \leq n,
$$

by Theorem 3.1. In other words, consider a polynomial ring

$$
\mathcal{P}:=\mathbf{Q}\left[t_{i}, t_{i_{1} i_{2}} \mid 1 \leq i \leq n, 1 \leq i_{1}<i_{2}<\leq n\right],
$$

and a ring homomorphism $\bar{\pi}=\bar{\pi}_{G}: \mathcal{P} \rightarrow \mathfrak{X}_{\mathbf{Q}}(G)$ defined by

$$
\bar{\pi}(1):=\frac{1}{2}\left(\operatorname{tr} 1_{G}\right), \quad \bar{\pi}\left(t_{i_{1} \cdots i_{l}}\right):=\operatorname{tr} x_{i_{1}} \cdots x_{i_{l}} .
$$

Then $\bar{\pi}: \mathcal{P} \rightarrow \mathfrak{X}_{\mathbf{Q}}(G)$ is surjective. Set $I:=\operatorname{Ker}(\bar{\pi})$. In this paper, we always identify $\mathcal{P} / I$ with $\mathfrak{X}_{\mathbf{Q}}(G)$ as a ring under the isomorphism induced from $\bar{\pi}$ and also call each of them the ring of Fricke characters of $G$ over $\mathbf{Q}$.

Next, set $t_{i_{1} \cdots i_{l}}^{\prime}:=t_{i_{1} \cdots i_{l}}-2 \in \mathcal{P}$. We also denote by $t_{i_{1} \cdots i_{l}}^{\prime}$ its coset class in $\mathcal{P} / I$ by abuse of language. An element in $\mathfrak{X}_{\mathbf{Q}}(G)$ corresponding to $t_{i_{1} \ldots i_{l}}^{\prime} \in \mathcal{P} / I$ is

$$
\begin{aligned}
\operatorname{tr}^{\prime} x_{i_{1}} \cdots x_{i_{l}}: & =\left(\operatorname{tr} x_{i_{1}} \cdots x_{i_{l}}\right)-2 \\
& =\left(\operatorname{tr} x_{i_{1}} \cdots x_{i_{l}}\right)-\operatorname{tr} 1_{G} \in \mathfrak{X}_{\mathbf{Q}}(G) .
\end{aligned}
$$

Consider an ideal

$$
J_{0}=\left(t_{i}^{\prime}, t_{i_{1} i_{2}}^{\prime} \mid 1 \leq i \leq n, 1 \leq i_{1}<i_{2} \leq n\right) \subset \mathcal{P}
$$

generated by all $t_{i_{1} \ldots i_{l}}^{\prime}$ 's in $\mathcal{P}$, and

$$
J:=\bar{\pi}\left(J_{0}\right)=\left(t_{i}^{\prime}, t_{i_{1} i_{2}}^{\prime} \mid 1 \leq i \leq n, 1 \leq i_{1}<i_{2} \leq n\right) \subset \mathcal{P} / I .
$$

Then, we have a descending filtration

$$
J \supset J^{2} \supset J^{3} \supset \cdots
$$

of ideals of $\mathcal{P} / I$. Set

$$
\operatorname{gr}^{k}(J):=J^{k} / J^{k+1}
$$


Then each of $\operatorname{gr}^{k}(J)$ is a finite dimensional Q-vector space. In the present paper, for the case where $G$ is a free abelian group $H$ of rank $n$, we determine the $\mathbf{Q}$-vector space structures of $\operatorname{gr}^{k}(J)$ for any $k \geq 1$. More precisely, we give a basis of each of $\operatorname{gr}^{k}(J)$ in Section 4 .

3.3. Basic formulae among $\operatorname{tr}^{\prime} x$. In this subsection, we summarize basic and useful formulae among $\operatorname{tr}^{\prime} x$ for $x \in G$. To begin with, we confirm the following.

$$
\begin{aligned}
& \operatorname{tr}^{\prime} x^{-1}=\operatorname{tr}^{\prime} x, \\
& \operatorname{tr}^{\prime} x y=\operatorname{tr}^{\prime} y x, \\
& \operatorname{tr}^{\prime} x y+\operatorname{tr}^{\prime} x y^{-1}=2 \operatorname{tr}^{\prime} x+2 \operatorname{tr}^{\prime} y+\left(\operatorname{tr}^{\prime} x\right)\left(\operatorname{tr}^{\prime} y\right),
\end{aligned}
$$

$$
\begin{aligned}
& \operatorname{tr}^{\prime} x y z+\operatorname{tr}^{\prime} y x z=-2\left\{\operatorname{tr}^{\prime} x+\operatorname{tr}^{\prime} y+\operatorname{tr}^{\prime} z\right\} \\
& \quad+2\left\{\operatorname{tr}^{\prime} x y+\operatorname{tr}^{\prime} y z+\operatorname{tr}^{\prime} x z\right\} \\
& \quad+\left(\operatorname{tr}^{\prime} x\right)\left(\operatorname{tr}^{\prime} y z\right)+\left(\operatorname{tr}^{\prime} y\right)\left(\operatorname{tr}^{\prime} x z\right)+\left(\operatorname{tr}^{\prime} z\right)\left(\operatorname{tr}^{\prime} x y\right), \\
& \quad-2\left\{\left(\operatorname{tr}^{\prime} x\right)\left(\operatorname{tr}^{\prime} y\right)+\left(\operatorname{tr}^{\prime} y\right)\left(\operatorname{tr}^{\prime} z\right)+\left(\operatorname{tr}^{\prime} z\right)\left(\operatorname{tr}^{\prime} x\right)\right\}-\left(\operatorname{tr}^{\prime} x\right)\left(\operatorname{tr}^{\prime} y\right)\left(\operatorname{tr}^{\prime} z\right),
\end{aligned}
$$

and

$$
\begin{aligned}
2 \operatorname{tr}^{\prime} x y z w= & 2\left(\operatorname{tr}^{\prime} x+\operatorname{tr}^{\prime} y+\operatorname{tr}^{\prime} z+\operatorname{tr}^{\prime} w\right) \\
& -2\left(\operatorname{tr}^{\prime} x y+\operatorname{tr}^{\prime} x z+\operatorname{tr}^{\prime} x w+\operatorname{tr}^{\prime} y z+\operatorname{tr}^{\prime} y w+\operatorname{tr}^{\prime} z w\right) \\
& +2\left(\operatorname{tr}^{\prime} x y z+\operatorname{tr}^{\prime} x y w+\operatorname{tr}^{\prime} x z w+\operatorname{tr}^{\prime} y z w\right) \\
& +2\left\{\left(\operatorname{tr}^{\prime} x\right)\left(\operatorname{tr}^{\prime} y\right)+\left(\operatorname{tr}^{\prime} x\right)\left(\operatorname{tr}^{\prime} w\right)\right. \\
& \left.+\left(\operatorname{tr}^{\prime} y\right)\left(\operatorname{tr}^{\prime} z\right)+\left(\operatorname{tr}^{\prime} z\right)\left(\operatorname{tr}^{\prime} w\right)+2\left(\operatorname{tr}^{\prime} x\right)\left(\operatorname{tr}^{\prime} z\right)+2\left(\operatorname{tr}^{\prime} y\right)\left(\operatorname{tr}^{\prime} w\right)\right\} \\
& -2\left\{\left(\operatorname{tr}^{\prime} x\right)\left(\operatorname{tr}^{\prime} y z\right)+\left(\operatorname{tr}^{\prime} x\right)\left(\operatorname{tr}^{\prime} z w\right)\right. \\
& +\left(\operatorname{tr}^{\prime} y\right)\left(\operatorname{tr}^{\prime} x w\right)+\left(\operatorname{tr}^{\prime} y\right)\left(\operatorname{tr}^{\prime} z w\right) \\
& +\left(\operatorname{tr}^{\prime} z\right)\left(\operatorname{tr}^{\prime} x y\right)+\left(\operatorname{tr}^{\prime} z\right)\left(\operatorname{tr}^{\prime} x w\right) \\
& \left.+\left(\operatorname{tr}^{\prime} w\right)\left(\operatorname{tr}^{\prime} x y\right)+\left(\operatorname{tr}^{\prime} w\right)\left(\operatorname{tr}^{\prime} y z\right)\right\} \\
& +\left\{\left(\operatorname{tr}^{\prime} x\right)\left(\operatorname{tr}^{\prime} y z w\right)+\left(\operatorname{tr}^{\prime} y\right)\left(\operatorname{tr}^{\prime} x z w\right)\right. \\
& \left.+\left(\operatorname{tr}^{\prime} z\right)\left(\operatorname{tr}^{\prime} x y w\right)+\left(\operatorname{tr}^{\prime} w\right)\left(\operatorname{tr}^{\prime} x y z\right)\right\}+\left\{\left(\operatorname{tr}^{\prime} x y\right)\left(\operatorname{tr}^{\prime} z w\right)\right. \\
& \left.-\left(\operatorname{tr}^{\prime} x z\right)\left(\operatorname{tr}^{\prime} y w\right)+\left(\operatorname{tr}^{\prime} x w\right)\left(\operatorname{tr}^{\prime} y z\right)\right\} \\
& -\left\{\left(\operatorname{tr}^{\prime} x\right)\left(\operatorname{tr}^{\prime} y\right)\left(\operatorname{tr}^{\prime} z w\right)+\left(\operatorname{tr}^{\prime} y\right)\left(\operatorname{tr}^{\prime} z\right)\left(\operatorname{tr}^{\prime} x w\right)\right.
\end{aligned}
$$




$$
\begin{aligned}
& \left.+\left(\operatorname{tr}^{\prime} x\right)\left(\operatorname{tr}^{\prime} w\right)\left(\operatorname{tr}^{\prime} y z\right)+\left(\operatorname{tr}^{\prime} z\right)\left(\operatorname{tr}^{\prime} w\right)\left(\operatorname{tr}^{\prime} x y\right)\right\} \\
& +\left(\operatorname{tr}^{\prime} x\right)\left(\operatorname{tr}^{\prime} y\right)\left(\operatorname{tr}^{\prime} z\right)\left(\operatorname{tr}^{\prime} w\right) \\
& +2\left\{\left(\operatorname{tr}^{\prime} x\right)\left(\operatorname{tr}^{\prime} y\right)\left(\operatorname{tr}^{\prime} z\right)+\left(\operatorname{tr}^{\prime} x\right)\left(\operatorname{tr}^{\prime} y\right)\left(\operatorname{tr}^{\prime} w\right)\right. \\
& \left.+\left(\operatorname{tr}^{\prime} x\right)\left(\operatorname{tr}^{\prime} z\right)\left(\operatorname{tr}^{\prime} w\right)+\left(\operatorname{tr}^{\prime} y\right)\left(\operatorname{tr}^{\prime} z\right)\left(\operatorname{tr}^{\prime} w\right)\right\}
\end{aligned}
$$

These formulae hold for any group $G$ and $x, y, z, w \in G$. For details, see our previous paper [5, Section 4].

Lemma 3.2. For any $x \in G$ and $\alpha \in \mathbf{Z}$,

$$
t r^{\prime} x^{\alpha} \equiv \alpha^{2} t r^{\prime} x \quad\left(\bmod J^{2}\right) .
$$

Proof. It is obvious when $\alpha=0,1$. It suffices to show $\alpha>0$. We show this by induction on $\alpha$.

Assume $\alpha \geq 2$. Then, substituting $x^{\alpha-1}$ and $x$ to $x$ and $y$ in (3.10), respectively, we have

$$
\operatorname{tr}^{\prime} x^{\alpha}+\operatorname{tr}^{\prime} x^{\alpha-2}=2 \operatorname{tr}^{\prime} x^{\alpha-1}+2 \operatorname{tr}^{\prime} x+\left(\operatorname{tr}^{\prime} x^{\alpha-1}\right)\left(\operatorname{tr}^{\prime} x\right),
$$

and, hence, by the inductive hypothesis, we obtain

$$
\operatorname{tr}^{\prime} x^{\alpha} \equiv 2(\alpha-1)^{2} \operatorname{tr}^{\prime} x-(\alpha-2)^{2} \operatorname{tr}^{\prime} x+2 \operatorname{tr}^{\prime} x, \equiv \alpha^{2} \operatorname{tr}^{\prime} x \quad\left(\bmod J^{2}\right) .
$$

This completes the proof of Lemma 3.2.

Here, we consider additional relations among $\operatorname{tr}^{\prime} x$ for abelian group $G$.

Lemma 3.3. For an abelian group $G$ and any $x, y, z, w \in G$, we have

$$
\begin{aligned}
& \left(t r^{\prime} x z\right)\left(\operatorname{tr}^{\prime} y w\right)=\left(t r^{\prime} x w\right)\left(t r^{\prime} y z\right) \\
& +\left\{\left(t r^{\prime} x\right)\left(t r^{\prime} z\right)+\left(t r^{\prime} y\right)\left(t r^{\prime} w\right)-\left(t r^{\prime} y\right)\left(t r^{\prime} z\right)-\left(t r^{\prime} x\right)\left(t r^{\prime} w\right)\right\} \\
& -\left\{\left(t r^{\prime} x\right)\left(t r^{\prime} y z\right)+\left(t r^{\prime} y\right)\left(t r^{\prime} x w\right)+\left(t r^{\prime} z\right)\left(t r^{\prime} x w\right)+\left(t r^{\prime} w\right)\left(t r^{\prime} y z\right)\right. \\
& \left.-\left(t r^{\prime} y\right)\left(t r^{\prime} x z\right)-\left(t r^{\prime} x\right)\left(t r^{\prime} y w\right)-\left(t r^{\prime} z\right)\left(t r^{\prime} y w\right)-\left(t r^{\prime} w\right)\left(t r^{\prime} x z\right)\right\} \\
& -\frac{1}{2}\left\{\left(t r^{\prime} y\right)\left(t r^{\prime} z\right)\left(t r^{\prime} x w\right)+\left(t r^{\prime} x\right)\left(t r^{\prime} w\right)\left(t r^{\prime} y z\right)\right. \\
& \left.-\left(t r^{\prime} x\right)\left(t r^{\prime} z\right)\left(t r^{\prime} y w\right)-\left(t r^{\prime} y\right)\left(t r^{\prime} w\right)\left(t r^{\prime} x z\right)\right\}
\end{aligned}
$$

Proof. In order to obtain the equation above, it suffices to calculate $2 \operatorname{tr}^{\prime} x y z w-2 \operatorname{tr}^{\prime} y x z w$ with equation (3.12). The calculation is straightforward. We leave it to the reader for an exercise. 
As special cases of Lemma 3.3, we see the following corollaries.

Corollary 3.4. For an abelian group $G$ and $x, y, z \in G$, we have

$$
\begin{aligned}
\left(\operatorname{tr}^{\prime} x y\right)\left(\operatorname{tr}^{\prime} x z\right)= & -3\left(\operatorname{tr}^{\prime} x\right)\left(\operatorname{tr}^{\prime} y\right)-3\left(\operatorname{tr}^{\prime} x\right)\left(\operatorname{tr}^{\prime} z\right)-\left(\operatorname{tr}^{\prime} x\right)^{2}-\left(\operatorname{tr}^{\prime} y\right)\left(\operatorname{tr}^{\prime} z\right) \\
& +2\left(\operatorname{tr}^{\prime} x\right)\left(\operatorname{tr}^{\prime} y z\right)+\left(\operatorname{tr}^{\prime} x\right)\left(\operatorname{tr}^{\prime} x y\right) \\
& +\left(\operatorname{tr}^{\prime} x\right)\left(\operatorname{tr}^{\prime} x z\right)+\left(\operatorname{tr}^{\prime} y\right)\left(\operatorname{tr}^{\prime} x z\right)+\left(\operatorname{tr}^{\prime} z\right)\left(\operatorname{tr}^{\prime} x y\right) \\
& +\frac{1}{2}\left(\operatorname{tr}^{\prime} x\right)^{2}\left(\operatorname{tr}^{\prime} y z\right)-\left(\operatorname{tr}^{\prime} x\right)^{2}\left(\operatorname{tr}^{\prime} y\right) \\
& -\left(\operatorname{tr}^{\prime} x\right)^{2}\left(\operatorname{tr}^{\prime} z\right)-\left(\operatorname{tr}^{\prime} y\right)\left(\operatorname{tr}^{\prime} x z\right) \\
& -\left(\operatorname{tr}^{\prime} x\right)\left(\operatorname{tr}^{\prime} y w\right)-\left(\operatorname{tr}^{\prime} z\right)\left(\operatorname{tr}^{\prime} y w\right)-\left(\operatorname{tr}^{\prime} w\right)\left(\operatorname{tr}^{\prime} x z\right) \\
& -\frac{1}{2}\left(\operatorname{tr}^{\prime} x\right)^{2}\left(\operatorname{tr}^{\prime} y\right)\left(\operatorname{tr}^{\prime} z\right)-2\left(\operatorname{tr}^{\prime} x\right)\left(\operatorname{tr}^{\prime} y\right)\left(\operatorname{tr}^{\prime} z\right) \\
& +\frac{1}{2}\left(\operatorname{tr}^{\prime} x\right)\left(\operatorname{tr}^{\prime} z\right)\left(\operatorname{tr}^{\prime} x y\right)+\frac{1}{2}\left(\operatorname{tr}^{\prime} x\right)\left(\operatorname{tr}^{\prime} y\right)\left(\operatorname{tr}^{\prime} x z\right)
\end{aligned}
$$

Corollary 3.5. For an abelian group $G$ and $x, y \in G$, we have

$$
\begin{aligned}
\left(t^{\prime} x y\right)^{2}= & -\left(t^{\prime} x\right)^{2}-\left(t^{\prime} y\right)^{2} \\
& +2\left\{\left(\operatorname{tr}^{\prime} x\right)\left(\operatorname{tr}^{\prime} y\right)+\left(\operatorname{tr}^{\prime} x\right)\left(t r^{\prime} x y\right)+\left(\operatorname{tr}^{\prime} y\right)\left(\operatorname{tr}^{\prime} x y\right)\right\} \\
& +\left(\operatorname{tr}^{\prime} x\right)\left(\operatorname{tr}^{\prime} y\right)\left(\operatorname{tr}^{\prime} x y\right) .
\end{aligned}
$$

4. The structure of a Q-vector space $\operatorname{gr}^{k}(J)$ for $G=H$. The goal of this section is to give a basis of $\operatorname{gr}^{k}(J)$ for any $k \geq 1$ as a Q-vector space.

Proposition 4.1. For any $k \geq 1$, consider a polynomial

$$
f:=t_{p_{1} q_{1}}^{\prime} \cdots t_{p_{l} q_{l}}^{\prime} t_{i_{l+1}}^{\prime} \cdots t_{i_{k}}^{\prime} \in \mathcal{P}
$$

for $0 \leq l \leq k,\left(p_{1}, q_{1}\right) \leq \cdots \leq\left(p_{l}, q_{l}\right)$ and $1 \leq i_{l+1} \leq \cdots \leq i_{k} \leq n$. Then, under the modulo $I$, the monomial $f$ can be written as a sum of monomials of type

$$
t_{p_{1}^{\prime} q_{1}^{\prime}}^{\prime} \cdots t_{p_{m}^{\prime} q_{m}^{\prime}}^{\prime} t_{j_{m+1}}^{\prime} \cdots t_{j_{k}}^{\prime} \in \mathcal{P}
$$

such that

- $0 \leq m \leq k$, 
- $1 \leq p_{1}^{\prime}<q_{1}^{\prime}<\cdots<p_{m}^{\prime}<q_{m}^{\prime} \leq n$,

- $1 \leq j_{m+1} \leq \cdots \leq j_{k} \leq n$,

- $\left\{p_{1}^{\prime}, q_{1}^{\prime}, \ldots, p_{m}^{\prime}, q_{m}^{\prime}\right\} \subset\left\{p_{1}, q_{1}, \ldots, p_{l}, q_{l}\right\}$.

Proof. We prove this proposition by induction on $l \geq 0$. If $l=0$ or 1 , it is clear. Assume $l \geq 2$.

First, assume that some elements in $\left\{p_{1}, q_{1}, \ldots, p_{l}, q_{l}\right\}$ are equal. For simplicity, we consider three cases:

(i) $p_{1}=p_{2}$ and $q_{1} \neq q_{2}$,

(ii) $p_{1}=q_{2}$ and $q_{1} \neq p_{2}$,

(iii) $p_{1}=p_{2}$ and $q_{1}=q_{2}$.

For parts (i) and (ii), by using Corollary 3.4, we see that under the modulo $I$, the monomial $f$ can be written as a sum of monomials

$$
t_{p_{1} q_{1}}^{\prime} \cdots t_{p_{r} q_{r}}^{\prime} t_{i_{r+1}}^{\prime} \cdots t_{i_{k}}^{\prime}
$$

such that $r<l$. Hence, by applying the inductive hypothesis to each of such monomials, we obtain the required result. We can discuss an argument similar to the above for case (iii) by using Corollary 3.5. Therefore, we assume that $p_{1}, q_{1}, \ldots, p_{l}, q_{l}$ are distinct.

Now, by the assumption $\left(p_{1}, q_{1}\right) \leq\left(p_{2}, q_{2}\right)$, we have $p_{1}<p_{2}$. If $q_{1}<p_{2}$, then we have the inequalities:

$$
\begin{aligned}
& p_{1}<q_{1}<p_{2}<p_{3}<\cdots<p_{l}
\end{aligned}
$$

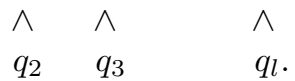

Hence, if we set $f^{\prime}:=t_{p_{2} q_{2}}^{\prime} \cdots t_{p_{l} q_{l}}^{\prime} t_{i_{l+1}}^{\prime} \cdots t_{i_{k}}^{\prime} \in \mathcal{P}$, then we can apply the inductive hypothesis to $f^{\prime}$ and obtain the required result.

Next, if $p_{2}<q_{1}$, we have the inequalities:

$$
\begin{aligned}
& p_{1}<p_{2}<p_{3}<\cdots<p_{l} \\
& \wedge_{q_{1}, q_{2}} \wedge q_{q_{3}} \quad \wedge_{q_{l} .}
\end{aligned}
$$

Then, by using Lemma 3.3, we can write $f$ as a sum of monomials

$$
t_{p_{1} p_{2}}^{\prime} t_{q_{1}, q_{2}}^{\prime} t_{p_{3} q_{3}}^{\prime} \cdots t_{p_{l} q_{l}}^{\prime} t_{i_{l+1}}^{\prime} \cdots t_{i_{k}}^{\prime}
$$


and

$$
t_{p_{1} q_{1}}^{\prime} \cdots t_{p_{r} q_{r}}^{\prime} t_{i_{r+1}}^{\prime} \cdots t_{i_{k}}^{\prime}
$$

such that $r<l$. If we apply the inductive hypothesis to $t_{q_{1}, q_{2}}^{\prime} t_{p_{3} q_{3}}^{\prime} \cdots$ $t_{p_{l} q_{l}}^{\prime} t_{i_{l+1}}^{\prime} \cdots t_{i_{k}}^{\prime}$ and $t_{p_{1} q_{1}}^{\prime} \cdots t_{p_{r} q_{r}}^{\prime} t_{i_{r+1}}^{\prime} \cdots t_{i_{k}}^{\prime}$, we obtain the required result. This completes the proof of Proposition 4.1.

Theorem 4.2. For each $k \geq 1$ and $0 \leq l \leq k$, set

$$
\begin{aligned}
T_{l}:= & \left\{t_{p_{1} q_{1}}^{\prime} \cdots t_{p_{l} q_{l}}^{\prime} t_{i_{l+1}}^{\prime} \cdots t_{i_{k}}^{\prime} \in J_{0}\right. \\
& \left.\mid 1 \leq p_{1}<q_{1}<\cdots<p_{l}<q_{l} \leq n, 1 \leq i_{l+1} \leq \cdots \leq i_{k} \leq n\right\} .
\end{aligned}
$$

Then

$$
S_{k}:=\bigcup_{l=0}^{k} \bar{\pi}\left(T_{l}\right)
$$

forms a basis of $g r^{k}(J)$.

Proof. By Proposition 4.1, we see that $S_{k}$ generates $\operatorname{gr}^{k}(J)$. In order to show the linear independence of the elements of $S_{k}$, set

$$
f:=\sum_{l=0}^{k} \sum_{\substack{p_{1}<q_{1}<\cdots<p_{l}<q_{l} \\ i_{l+1} \leq \cdots \leq i_{k}}} a_{p_{1} q_{1}, \ldots, p_{l} q_{l}, i_{l+1}, \ldots, i_{k}} t_{p_{1} q_{1}}^{\prime} \cdots t_{p_{l} q_{l}}^{\prime} t_{i_{l+1}}^{\prime} \cdots t_{i_{k}}^{\prime} \in J_{0}^{k},
$$

for $a_{p_{1} q_{1}, \ldots, p_{l} q_{l}, i_{l+1}, \ldots, i_{k}} \in \mathbf{Q}$, and assume $\bar{\pi}(f) \in J^{k+1}$.

Consider the interior

$$
D:=\{z \in \mathbf{C} \mid z \bar{z}<1\}
$$

of the unit disk in $\mathbf{C}$. For any $s_{1}, \ldots, s_{n} \in D$, we define a representation $\rho: H \rightarrow \mathrm{SL}(2, \mathbf{C})$ by

$$
\rho\left(x_{i}\right):=\left(\begin{array}{cc}
1-s_{i} & 0 \\
0 & \left(1-s_{i}\right)^{-1}
\end{array}\right)
$$

for any $1 \leq i \leq n$. If we consider the power series expansion

$$
\frac{1}{1-s_{i}}=1+s_{i}+s_{i}^{2}+s_{i}^{3}+\cdots
$$


at the origin on $D$, we can write each of $\operatorname{tr}^{\prime} \rho\left(x_{i}\right)$ and $\operatorname{tr}^{\prime} \rho\left(x_{i} x_{j}\right)$ as a convergent power series:

$$
\begin{aligned}
\operatorname{tr}^{\prime} \rho\left(x_{i}\right) & =\frac{s_{i}^{2}}{1-s_{i}}=s_{i}^{2}+s_{i}^{3}+s_{i}^{4}+\cdots, \\
\operatorname{tr}^{\prime} \rho\left(x_{i} x_{j}\right) & =s_{i}^{2}+2 s_{i} s_{j}+s_{j}^{2}+(\text { terms of degree } \geq 3) .
\end{aligned}
$$

Then we have

$$
\begin{gathered}
\bar{\pi}(f)(\rho)=\sum_{l=0}^{k} \sum_{\substack{p_{1}<q_{1}<\cdots<p_{l}<q_{l} \\
i_{l+1} \leq \cdots \leq i_{k}}}\left\{a_{p_{1} q_{1}, \ldots, p_{l} q_{l}, i_{l+1}, \ldots, i_{k}}\left(s_{p_{1}}^{2}+2 s_{p_{1}} s_{q_{1}}+s_{q_{1}}^{2}\right) \cdots\right. \\
\left.\left(s_{p_{2}}^{2}+2 s_{p_{2}} s_{q_{2}}+s_{q_{2}}^{2}\right) s_{i_{l+1}}^{2} \cdots s_{k}^{2}\right\} \\
+(\text { terms of degree } \geq 2 k+2) .
\end{gathered}
$$

Since $\bar{\pi}(f) \in J^{k+1}$, if we regard $\bar{\pi}(f)(\rho)$ as a polynomial on $s_{i}$ s, its degree must be greater than or equal to $2 k+2$. Hence, all coefficients of degree $2 k$ are zero.

To begin with, for any $1 \leq p_{1}<q_{2}<\cdots<p_{k}<q_{k} \leq n$, by observing the coefficients of $s_{p_{1}} s_{q_{1}} \cdots s_{p_{k}} s_{q_{k}}$, we see $a_{p_{1} q_{1}, \ldots, p_{k} q_{k}}=0$. For any $0 \leq l \leq k$, assume that

$$
a_{p_{1} q_{1}, \ldots, p_{m} q_{m}, i_{m+1}, \ldots, i_{k}}=0
$$

for any $l \leq m$. Then, for any $1 \leq p_{1}<q_{2}<\cdots<p_{l-1}<q_{l-1} \leq n$ and $1 \leq i_{l} \leq \cdots \leq i_{k} \leq n$, we see

$$
a_{p_{1} q_{1}, \ldots, p_{l-1} q_{l-1}, i_{l}, \ldots, i_{k}}=0 .
$$

Therefore, by the inductive argument, we verify that all coefficients of $f$ are equal to zero. This shows that elements in $S_{k}$ are linearly independent. This completes the proof of Theorem 4.2.

By observing the proof of Theorem 4.2, we have the following.

Theorem 4.3. The ideal I is generated by

$$
\begin{aligned}
t_{i r}^{\prime} t_{j s}^{\prime} & -t_{i s}^{\prime} t_{j r}^{\prime}-\left\{t_{i}^{\prime} t_{r}^{\prime}+t_{j}^{\prime} t_{s}^{\prime}-t_{j}^{\prime} t_{r}^{\prime}-t_{i}^{\prime} t_{s}^{\prime}\right\} \\
& +\left\{t_{i}^{\prime} t_{j r}^{\prime}+t_{j}^{\prime} t_{i s}^{\prime}+t_{r}^{\prime} t_{i s}^{\prime}+t_{s}^{\prime} t_{j r}^{\prime}-t_{j}^{\prime} t_{i r}^{\prime}-t_{i}^{\prime} t_{j s}^{\prime}-t_{r}^{\prime} t_{j s}^{\prime}-t_{s}^{\prime} t_{i r}^{\prime}\right\} \\
& +\frac{1}{2}\left\{t_{j}^{\prime} t_{r}^{\prime} t_{i s}^{\prime}+t_{i}^{\prime} t_{s}^{\prime} t_{j r}^{\prime}-t_{i}^{\prime} t_{r}^{\prime} t_{j s}^{\prime}-t_{j}^{\prime} t_{s}^{\prime} t_{i r}^{\prime}\right\}
\end{aligned}
$$


for any $1 \leq i, j, r, s \leq n$. Here we remark that, in the above notation, $t_{i j}^{\prime}$ should be read

$$
\begin{cases}t_{j i}^{\prime} & \text { if } i>j, \\ \left(t_{i}^{\prime}\right)^{2}+4 t_{i}^{\prime} & \text { if } i=j .\end{cases}
$$

In particular, I is finitely generated.

Proof. Let $I^{\prime}$ be an ideal of $\mathcal{P}$ generated by elements (4.1). From Lemma 3.3, we have $I^{\prime} \subset I$. For any $f \in I$, observing the proof of Proposition 4.1, we see that $f$ can be written as

$$
\begin{gathered}
f \equiv \sum_{k \geq 0} \sum_{l=0}^{k} \sum_{\substack{p_{1}<q_{1}<\cdots<p_{l}<q_{l} \\
i_{l+1} \leq \cdots \leq i_{k}}} a_{p_{1} q_{1}, \ldots, p_{l} q_{l}, i_{l+1}, \ldots, i_{k}} t_{p_{1} q_{1}}^{\prime} \cdots \\
t_{p_{l} q_{l}}^{\prime} t_{i_{l+1}}^{\prime} \cdots t_{i_{k}}^{\prime} \in J_{0}^{k}
\end{gathered}
$$

modulo $I^{\prime}$. Here, in the sum of the right hand side of the equation above, $k$ runs over finitely many non-negative integers. Hence, by Theorem 4.2, we see that all coefficients of $f$ are zero and obtain $f \in I^{\prime}$. This shows $I=I^{\prime}$.

Remark 4.4. We remark that, if $n=1$, we have $I=(0)$.

\section{Theorem 4.5 .}

$$
\bigcap_{k \geq 1} J^{k}=\{0\}
$$

Proof. For any $f \in \bigcap_{k>1} J^{k}$, we can write $f$ as (4.2). Then, observing the coset class of $f$ in $\operatorname{gr}^{1}(J)$, we see that $a_{p_{1} q_{1}}=a_{i_{1}}=0$ for any $1 \leq p_{1}<q_{1} \leq n$ and $1 \leq i_{1} \leq n$. Next, observing the coset class of $f$ in $\operatorname{gr}^{2}(J)$, we see that all coefficients of $f$ of degree 2 are zero. By repeating this argument inductively, we obtain $f=0$. This completes the proof of Theorem 4.5.

5. The primeness of the ideal $I$. In this section, we show that the ideal $I$ is a prime ideal of $\mathcal{P}$; in other words, $\mathcal{P} / I$ is an integral domain. In order to show this, we introduce a weight of an element $f \in \mathcal{P} / I$. From Theorem 4.5, for any $f \in \mathcal{P} / I \backslash\{0\}$, there exists some 
integer $k \geq 0$ such that $f \in J^{k} \backslash J^{k+1}$. Then we call $k$ the weight of $f$, and denote it by wt $(f)$.

Proposition 5.1. For any $f, g \in \mathcal{P} / I \backslash\{0\}$, wt $(f g)=$ wt $(f)+$ wt $(g)$.

Proof. It is obvious if wt $(f)=0$ or wt $(g)=0$. Hence, we may assume wt $(f)$, wt $(g) \geq 1$. Set $k_{1}:=$ wt $(f)$ and $k_{2}:=\operatorname{wt}(g)$. Since it is clear that wt $(f g) \geq k_{1}+k_{2}$, assume wt $(f g)>k_{1}+k_{2}$.

For any integers $\alpha_{1}, \ldots, \alpha_{n} \in \mathbf{Z}$, consider a group homomorphism $\rho_{\left(\alpha_{1}, \ldots, \alpha_{n}\right)}: H \rightarrow \mathbf{Z}=\left\langle x_{1}\right\rangle$ defined by $x_{i} \mapsto x_{1}^{\alpha_{i}}$ for any $1 \leq i \leq n$. Then $\rho_{\left(\alpha_{1}, \ldots, \alpha_{n}\right)}$ induces a ring homomorphism $\bar{\rho}_{\left(\alpha_{1}, \ldots, \alpha_{n}\right)}: \mathfrak{X}_{\mathbf{Q}}(H) \rightarrow \mathfrak{X}_{\mathbf{Q}}(\mathbf{Z})$ defined by

$$
\operatorname{tr}^{\prime} x \longmapsto \operatorname{tr}^{\prime} \rho_{\left(\alpha_{1}, \ldots, \alpha_{n}\right)}(x)
$$

Namely, we have

$$
\bar{\rho}_{\left(\alpha_{1}, \ldots, \alpha_{n}\right)}\left(\operatorname{tr}^{\prime} x_{i}\right)=\operatorname{tr}^{\prime} x_{1}^{\alpha_{i}}, \quad \bar{\rho}_{\left(\alpha_{1}, \ldots, \alpha_{n}\right)}\left(\operatorname{tr}^{\prime} x_{i} x_{j}\right)=\operatorname{tr}^{\prime} x_{1}^{\alpha_{i}+\alpha_{j}},
$$

Now, set

$$
\begin{aligned}
& f:=\sum_{l=0}^{k_{1}} \sum_{\substack{p_{1}<q_{1}<\cdots<p_{l}<q_{l} \\
i_{l+1} \leq \cdots \leq i_{k_{1}}}} a_{p_{1} q_{1}, \ldots, p_{l} q_{l}, i_{l+1}, \ldots, i_{k_{1}}} t_{p_{1} q_{1}}^{\prime} \ldots \\
& t_{p_{l} q_{l}}^{\prime} t_{i_{l+1}}^{\prime} \cdots t_{i_{k_{1}}}^{\prime}+\left(\text { terms of degree }>k_{1}\right), \\
& g:=\sum_{\substack{m=0 \\
p_{1}^{\prime}<q_{1}^{\prime}<\cdots<p_{m}^{\prime}<q_{m}^{\prime} \\
i_{m+1}^{\prime} \leq \cdots \leq i_{k_{2}}^{\prime}}} \sum_{p_{1}^{\prime} q_{1}^{\prime}, \ldots, p_{m}^{\prime} q_{m}^{\prime}, i_{m+1}^{\prime}, \ldots, i_{k_{2}}^{\prime}} t_{p_{1}^{\prime} q_{1}^{\prime}}^{\prime} \cdots \\
& t_{p_{m}^{\prime} q_{m}^{\prime}}^{\prime} t_{i_{m+1}^{\prime}}^{\prime} \cdots t_{i_{k_{2}}^{\prime}}^{\prime}+\left(\text { terms of degree }>k_{2}\right) \text {, }
\end{aligned}
$$

and set $F:=\bar{\rho}_{\left(\alpha_{1}, \ldots, \alpha_{n}\right)}(f)$ and $G:=\bar{\rho}_{\left(\alpha_{1}, \ldots, \alpha_{n}\right)}(g)$. Then wt $(F G)>$ $k_{1}+k_{2}$ in $\mathfrak{X}_{\mathbf{Q}}(\mathbf{Z})$. Hence, the coefficient $P\left(\alpha_{1}, \ldots, \alpha_{n}\right)$ of $F G$ of degree $k_{1}+k_{2}$ is equal to zero. Here, using Lemma 3.2, we have $P\left(\alpha_{1}, \ldots, \alpha_{n}\right)=P_{1} P_{2}$ for

$$
\begin{array}{r}
P_{1}:=\left(\sum_{l=0}^{k_{1}} \sum_{\begin{array}{r}
p_{1}<q_{1}<\cdots<p_{l}<q_{l} \\
i_{l+1} \leq \cdots \leq i_{k_{1}}
\end{array}} a_{p_{1} q_{1}, \ldots, p_{l} q_{l}, i_{l+1}, \ldots, i_{k_{1}}}\left(\alpha_{p_{1}}+\alpha_{q_{1}}\right)^{2} \cdots\right. \\
\left.\left(\alpha_{p_{l}}+\alpha_{q_{l}}\right)^{2} \alpha_{i_{l+1}}^{2} \cdots \alpha_{i_{k_{1}}}^{2}\right)
\end{array}
$$




$$
\begin{array}{r}
P_{2}:=\left(\sum_{\substack{m=0 \\
p_{1}^{\prime}<q_{1}^{\prime}<\cdots<p_{m}^{\prime}<q_{m}^{\prime} \\
i_{m+1}^{\prime} \leq \cdots \leq i_{k_{2}}^{\prime}}} a_{p_{1}^{\prime} q_{1}^{\prime}, \ldots, p_{m}^{\prime} q_{m}^{\prime}, i_{m+1}^{\prime}, \ldots, i_{k_{2}}^{\prime}}\left(\alpha_{p_{1}^{\prime}}+\alpha_{q_{1}^{\prime}}\right)^{2} \ldots\right. \\
\left.\left(\alpha_{p_{m}^{\prime}}+\alpha_{q_{m}^{\prime}}\right)^{2} \alpha_{i_{m+1}^{\prime}}^{2} \cdots \alpha_{i_{k_{2}}^{\prime}}^{2}\right) .
\end{array}
$$

Consider $P\left(\alpha_{1}, \ldots, \alpha_{n}\right)$ as a polynomial in $\mathbf{Q}\left[\alpha_{1}, \ldots, \alpha_{n}\right]$. Since $P\left(\alpha_{1}, \ldots, \alpha_{n}\right)=0$ if $\alpha_{1}, \ldots, \alpha_{n}$ runs over all integers, we see that $P\left(\alpha_{1}, \ldots, \alpha_{n}\right)=0$ as a polynomial in $\mathbf{Q}\left[\alpha_{1}, \ldots, \alpha_{n}\right]$. Since $\mathbf{Q}\left[\alpha_{1}, \ldots, \alpha_{n}\right]$ is a domain, we have $P_{1}=0$ or $P_{2}=0$ in $\mathbf{Q}\left[\alpha_{1}, \ldots, \alpha_{n}\right]$.

Assume $P_{1}=0$. Then each coefficient of monomials in $\alpha_{1}, \ldots, \alpha_{n}$ in $P_{1}$ is equal to zero. First, by observing the coefficient of $\alpha_{p_{1}} \alpha_{q_{1}} \ldots$ $\alpha_{p_{k_{1}}} \alpha_{q_{k_{1}}}$, we see

$$
a_{p_{1} q_{1}, \ldots, p_{k_{1}} q_{k_{1}}}=0 .
$$

Furthermore, by an argument similar to that in the proof of Theo-

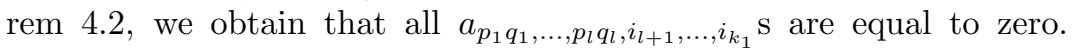
Hence, $f \in J^{k_{1}+1}$. This contradicts wt $(f)=k_{1}$. By the same argument, if $P_{2}=0$, we have $g \in J^{k_{2}+1}$, and a contradiction. Thus, we conclude that wt $(f g)=k_{1}+k_{2}$. This completes the proof of Proposition 5.1.

As a corollary, we obtain

Theorem 5.2. The quotient ring $\mathcal{P} / I$ is an integral domain. That is, the ideal $I$ is a prime ideal in $\mathcal{P}$.

6. Some remarks. Finally, we give some remarks about an action of the automorphism group Aut $H$ of $H$ on the ring $\mathfrak{X}_{\mathbf{Q}}(H)$. In general, for any group $G$, the automorphism group Aut $G$ of $G$ naturally acts on $\mathfrak{X}_{\mathbf{Q}}(G)$ from the right. (See our previous paper [5] for details.) In particular, for any $\sigma \in$ Aut $G$ and $x \in G$, the action of $\sigma \in$ Aut $G$ on $\operatorname{tr}^{\prime} x \in \mathfrak{X}_{\mathbf{Q}}(G)$ is given by

$$
\left(\operatorname{tr}^{\prime} x\right)^{\sigma}=\operatorname{tr}^{\prime} x^{\sigma}
$$

Clearly, we see that the ideal $J$ generated by all $\operatorname{tr}^{\prime} x$ for $x \in G$ is an Aut $G$-invariant ideal of $\mathfrak{X}_{\mathbf{Q}}(G)$. Hence, Aut $G$ naturally acts on $\operatorname{gr}^{k}(J)$ for each $k \geq 1$. 
Let $\mathcal{E}_{G}(k)$ be the kernel of a natural homomorphism Aut $G \rightarrow$ Aut $\left(J / J^{k+1}\right)$ induced from the action of Aut $G$. Then the groups $\mathcal{E}_{G}(k)$ define a descending filtration

$$
\mathcal{E}_{G}(1) \supset \mathcal{E}_{G}(2) \supset \cdots \supset \mathcal{E}_{G}(k) \supset \cdots
$$

of Aut $G$. In [5], we have shown that this filtration is central. That is, $\left[\mathcal{E}_{G}(k), \mathcal{E}_{G}(l)\right] \subset \mathcal{E}_{G}(k+l)$ for any $k, l \geq 1$. This is a Fricke character analogue of the Andreadakis-Johnson filtration of Aut $G$. (For details on the Andreadakis-Johnson filtration, see $[\mathbf{1 5}, \mathbf{1 6}]$, for example.)

Here, we determine $\mathcal{E}_{H}(1)$ and show $\mathcal{E}_{H}(k)=\mathcal{E}_{H}(1)$ for any $k \geq 1$. Let $\iota \in$ Aut $H$ be an automorphism of $H$ defined by

$$
x_{i}^{\iota}:=x_{i}^{-1}, \quad 1 \leq i \leq n .
$$

Then we have

Proposition 6.1. $\mathcal{E}_{H}(1)=\langle\iota\rangle$. Namely, $\mathcal{E}_{H}(1)$ is the cyclic group of order 2 , generated by $\iota$.

Proof. Clearly, we see $\mathcal{E}_{H}(1) \supset\langle\iota\rangle$. For any $\sigma \in \mathcal{E}_{H}(1)$, set

$$
x_{i}^{\sigma}:=x_{1}^{e_{1}(i)} x_{2}^{e_{2}(i)} \cdots x_{n}^{e_{n}(i)}, \quad 1 \leq i \leq n .
$$

It suffices to show

- $e_{j}(i)=0$ if $j \neq i$,

- $e_{1}(1)=\cdots=e_{n}(n)= \pm 1$.

For any $1 \leq i \leq n$ and any $s_{i} \in D$, consider a homomorphism $\rho_{i}: H \rightarrow \mathrm{SL}(2, \mathbf{C})$, defined by

$$
\rho_{i}\left(x_{j}\right):=\left\{\begin{array}{cc}
\left(\begin{array}{cc}
1-s_{i} & 0 \\
0 & \left(1-s_{i}\right)^{-1}
\end{array}\right), & \text { if } j=i, \\
E_{2}, & \text { if } j \neq i .
\end{array}\right.
$$

Then, from

$$
\operatorname{tr}^{\prime} x_{i} \equiv \operatorname{tr}^{\prime} x_{i}^{\sigma}=\operatorname{tr}^{\prime} x_{1}^{e_{1}(i)} x_{2}^{e_{2}(i)} \cdots x_{n}^{e_{n}(i)}\left(\bmod J^{2}\right),
$$

by substituting $\rho_{i}$, we see

$$
s_{i}^{2} \equiv e_{i}(i)^{2} s_{i}^{2} \quad\left(\bmod \left(s_{i}^{3}\right)\right),
$$


and hence $e_{i}(i)= \pm 1$. On the other hand, by substituting $\rho_{j}$ for $j \neq i$, we see

$$
0 \equiv e_{j}(i)^{2} s_{j}^{2} \quad\left(\bmod \left(s_{j}^{3}\right)\right)
$$

and hence $e_{j}(i)=0$.

In order to show $e_{1}(1)=\cdots=e_{n}(n)$, assume that $e_{i}(i) \neq e_{j}(j)$ for some $i$ and $j$. This means $\left(e_{i}(i), e_{j}(j)\right)=( \pm 1, \mp 1)$. For any $s_{i}, s_{j} \in D$, consider a homomorphism $\rho_{i j}: H \rightarrow \mathrm{SL}(2, \mathbf{C})$ defined by

$$
\rho_{i j}\left(x_{r}\right):= \begin{cases}\rho_{r}\left(x_{r}\right), & \text { if } r=i, j \\ E_{2}, & \text { if otherwise. }\end{cases}
$$

Then, by substituting $\rho_{i j}$ for equation $\operatorname{tr}^{\prime} x_{i} x_{j} \equiv \operatorname{tr}^{\prime}\left(x_{i} x_{j}\right)^{\sigma}=\operatorname{tr}^{\prime} x_{i}^{e_{i}(i)}$ $x_{j}^{e_{j}(j)}\left(\bmod J^{2}\right)$, we obtain

$$
s_{i}^{2}+2 s_{i} s_{j}+s_{j}^{2} \equiv s_{i}^{2}-2 s_{i} s_{j}+s_{j}^{2} \quad\left(\bmod \left(s_{i}^{3}, s_{i}^{2} s_{j}, s_{i} s_{j}^{2}, s_{j}^{3}\right)\right) .
$$

This is a contradiction. Therefore, we obtain the required result. This completes the proof of Proposition 6.1.

Corollary 6.2. For any $k \geq 2, \mathcal{E}_{H}(k)=\mathcal{E}_{H}(1)$.

Proof. In general, we have $\mathcal{E}_{H}(k) \subset \mathcal{E}_{H}(1)$. On the other hand, $\mathcal{E}_{H}(k) \supset \mathcal{E}_{H}(1)$ immediately follows from Proposition 6.1.

\section{REFERENCES}

1. F.G.-Acuna, J. Maria and Montesinos-Amilibia, On the character variety of group representations in $S L(2, \mathbf{C})$ and $P S L(2, \mathbf{C})$, Math. Z. 214 (1993), 627-652.

2. S. Andreadakis, On the automorphisms of free groups and free nilpotent groups, Proc. London Math. Soc. 15 (1965), 239-268.

3. R. Fricke and F. Klein, Vorlesungen über die Teorie der automorphen Functionen, Volume 1, B.G. Teuber (1897), 365-370.

4. M. Hall, The theory of groups, Chelsea Publishing Company, New York, 1976.

5. E. Hatakenaka and T. Satoh, On the graded quotients of the ring of Fricke characters of a free group, arXiv: 1206.1500 .

6. R. Horowitz, Characters of free groups represented in the two-dimensional special linear group, Comm. Pure Appl. Math. 25 (1972), 635-649.

7. , Induced automorphisms on Fricke characters of free groups, Trans. Amer. Math. Soc. 208 (1975), 41-50. 
8. D. Johnson, An abelian quotient of the mapping class group, Math. Ann. 249 (1980), 225-242.

9. $\longrightarrow$ The structure of the Torelli group I: A finite set of generators for $\mathcal{I}$, Ann. Math. 118 (1983), 423-442.

10. _ The structure of the Torelli group II: A characterization of the group generated by twists on bounding curves, Topology 24 (1985), 113-126.

11. The structure of the Torelli group III: The abelianization of $\mathcal{I}$, Topology 24 (1985), 127-144.

12. C. Maclachlan and A.W. Reid, The arithmetic of hyperbolic 3-manifolds, Grad. Texts Math. 219, Springer, New York, 2003.

13. W. Magnus, Rings of Fricke characters and automorphism groups of free groups, Math. Z. 170 (1980), 91-103.

14. S. Morita, Abelian quotients of subgroups of the mapping class group of surfaces, Duke Math. J. 70 (1993), 699-726.

15. T. Satoh, The cokernel of the Johnson homomorphisms of the automorphism group of a free metabelian group, Trans. Amer. Math. Soc. 361 (2009), 2085-2107.

16. _ A survey of the Johnson homomorphisms of the automorphism groups of free groups and related topics, in Handbook of Teichmüller theory, A. Papadopoulos, ed., arXiv:1204.0876.

17. H. Vogt, Sur les invariants fondamentaux des équations différentielles linéaires du second ordre, Ann. Sci. Ecol. Norm. Sup. 6 (1889), 3-72.

18. A. Whittemore, On special linear characters of free groups of rank $n \geq 4$, Proc. Amer. Math. Soc. 40 (1973), 383-388.

Tokyo University of Agriculture and Technology, 2-24-16, NakA-Cho, KOGANEI-SHI, TOKYO, 184-8588, JAPAN

Email address: hataken@cc.tuat.ac.jp

Department of Mathematics, Faculty of Science Division II, Tokyo UniverSity of Science, 1-3 Kagurazaka, Shinjuku, Tokyo, 162-8601, Japan

Email address: takao@rs.tus.ac.jp 